\title{
Antenna Selection for MIMO with Reduced Complexity
}

\author{
Deepika Patil, B. Suryakanth
}

\begin{abstract}
In this paper the imperfect calculation is connected, in both transmitter and beneficiary side displays a similar limit execution for various SNRs. These qualities get from this paper gives a correct problematic radio wire choice for transmitter side just as collector side re-enactment and looked at.
\end{abstract}

Keywords: MIMO system, Antenna selection, Sub-optimal technique, optimal technique

\section{INTRODUCTION}

$\mathrm{D}$ ifferent information various output(MIMO) remote frameworks, includes the numerous reception apparatus at transmitter side as collector side, have shown the capacity for expanding limit in high multipath situations, no adjustments in data transmission or transmitted power [1-3], henceforth this system is future for remote correspondence . in this strategy the approaching information is divided into sub streams and after that transmitted through various receiving wire to get the high ghastly productivity, however in MIMO required same number of RF chains as quite a bit of reception apparatus this causes mind-boggling expense and multifaceted nature . To over originate from this issue is reception apparatus choice method .This implementation of radio wire choice advanced flag preparing is impossible other procedure receiving wire determination turn into an alluring alternative [4]. Ideal receiving wire choice strategy is greater unpredictability and time required is more as review on this paper [5, 6, and 9] the methodology going for diminishing computational intricacy a progression of streamlined radio procedure for MIMO framework is great.

\section{SYSTEM MODEL}

Fig. 1: demonstrates a square chart of Antenna choice MIMO correspondence framework shows involving a transmitter TX and receiver $\mathrm{Rx}$. The transmitter Tx contains a transmission baseband unit, NT number of RF chains units, and M number of receiving wires The beneficiary RX includes NR number of reception apparatuses here a radio wire determination unit chose lists, the input data is sent from the chose records [10] Consider a N_R $\times$ N_T MIMO framework with N_T transmit receiving wire $\mathrm{N}_{\text {R }}$ Receive radio wire here $\mathrm{M}\left(\mathrm{M} \leq \mathrm{N} \_\mathrm{R}\right)$

As appeared in fig .1. For most extreme limit we select $M$ $\left(\mathrm{M} \leq \mathrm{N} \_\mathrm{R}\right)$ out for flag handling Info yield connection in MIMO framework is given by

Revised Manuscript Received on December 12, 2019

Deepika Patil, Assistant Professor, Department of ECE, MRECW Hyderabad, India. E-mail: deepikapati1021@gmail.com

B. Suryakanth, Professor, Department of ECE, BKIT Bhalki, Karnataka, India. E-mail: bsuryakanth3413@gmail.com wire choice algorithms in that Sub-ideal Antenna choice

Is a N_T $\times 1$ transmitted flag vector, $\rho$ is the normal flag to clamour proportion $(\mathrm{SNR})$ at get reception apparatus $x=\sqrt{\frac{\rho}{N_{T}} H_{g}+n_{x}}$

Where, $x=\left\lceil x 1, x 2, \ldots \ldots \ldots x_{\mathbb{N}_{R}}\right]^{T}$ is a $N_{R} \times 1$ received signal vector? $s=\left\lceil S 1, S 2 \ldots \ldots . . S_{\mathbb{N}_{T}}\right\rceil^{T}$

Is an $N_{T} \times 1$ transmitted signal vector, $\rho$ is the average signal to noise ratio (SNR) at receive antenna

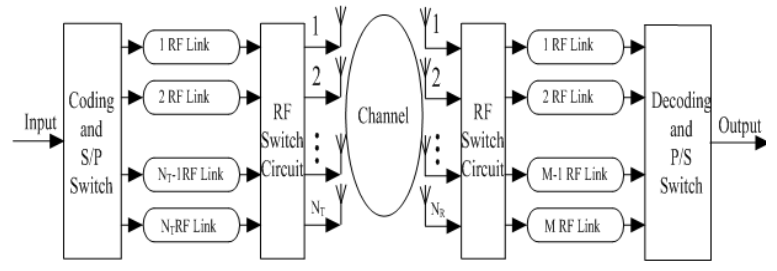

Fig. 1: Antenna selection MIMO communication system model.

\section{SUB-OPTIMAL ANTENNA SELECTION}

So as to lessen its multifaceted nature, we may need to fall back on the problematic strategy. For instance, extra reception apparatus can be chosen in rising request of expanding the channel limit. All the more explicitly, one reception apparatus with the most elevated limit is first chosen as

$$
\begin{aligned}
& R_{1}^{\text {gubopt }}=
\end{aligned}
$$

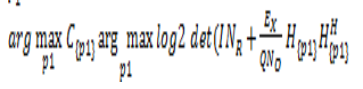

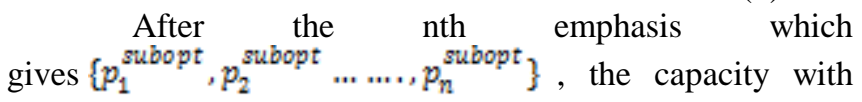
added antenna, equation 1 written as

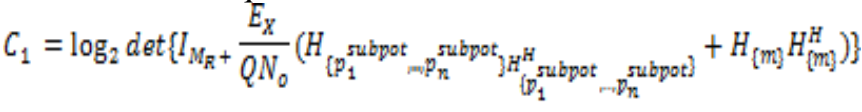

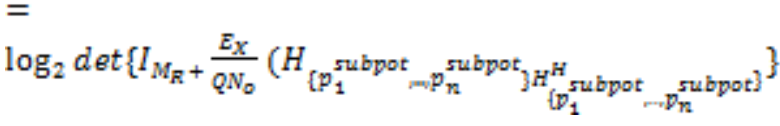

$$
\begin{aligned}
& \log _{2}\{1+
\end{aligned}
$$

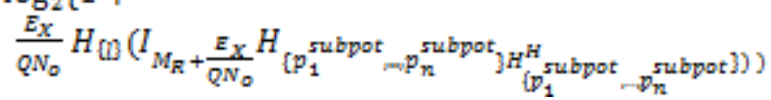

The addition $(n+1)^{\text {the }}$ antenna is the one increases the channel capacity in equation (3), that is,

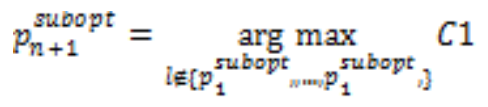

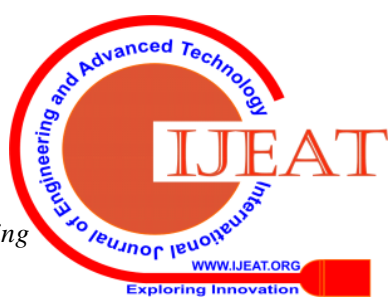




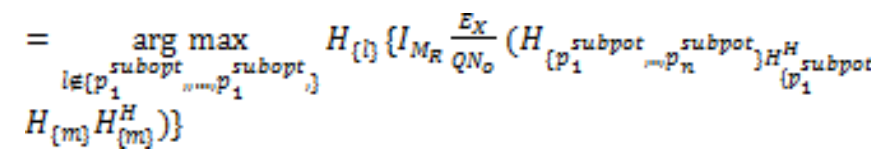

This procedure proceeds until all $\mathrm{M}$ radio wires are chosen (for example proceed with the cycle condition (4) until $\mathrm{n}+1=\mathrm{M})$. Note just a single grid reversal is require for all $M \in\left\{1,2, \ldots \ldots, N_{T}\right\}-\left\{p_{1}^{\text {subopt }}{ }_{, \ldots \ldots \ldots} p_{n}^{\text {subopt }}\right\}$ in the course of the selection process.

Meanwhile, a similar procedure can be executed by erasing the reception apparatus in sliding request of diminishing channel limit. Let son indicates set of reception apparatus records in the nth cycle. In the underlying step, we consider all antennas, $S_{-} 1=\left\{1,2, N_{-} T\right\}$, and selected the radio wire that utilizes in any event the capacity, that is,

$$
\begin{aligned}
& p_{1}^{\text {deleted }}= \\
& \underset{p_{1} \varepsilon_{1}}{\arg \max } \log _{2} \operatorname{det}\left(I_{M_{R}}+\frac{E_{X}}{Q N_{Q}} H_{s_{1}\left[\eta_{1}\right]} H_{r_{1}}^{H}\right. \text { (p1) }
\end{aligned}
$$

The antenna selected from equation (5) will be removed from the antenna index set, and the leftover antenna set is updated to $S_{2}=S_{1}-\left\{p_{1}^{\text {deleted }}\right\}$.If $\left|S_{2}\right|=N_{M}-1>L$, we pick another reception apparatus to erase. This will be the one that constributes slightest to the limit now for the present recieving wire list set $S_{-}(2$, ) that is,

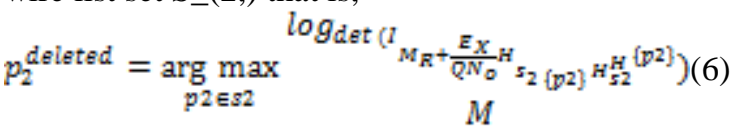

Again, the remaining antenna index set is given to $s_{\mathrm{a}}=s_{2}-\left\{p_{2}^{\text {deleted }}\right\}$, by considering the execution of problematic choice strategy, here chooses the radio wire subset and augmenting the channel limit

\section{RESULT AND DISCUSION}

Here we thought about the N_T=N_R=4, at lower SNR reception apparatus 3 and 4 is steady for all SNR esteems, radio wire 3 is great limit maker at higher SNR receiving wire 4 is great by knowing this as receiving wire increment at both the side the problematic reception apparatus choice give great limit as appeared in fig

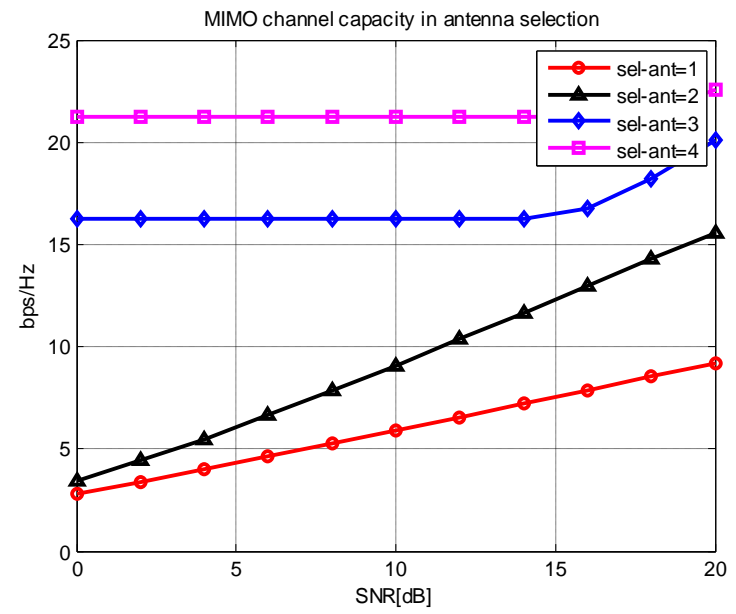

Fig.2: Capacity based Sub-optimal technique for $4 X 4$ MIMO system

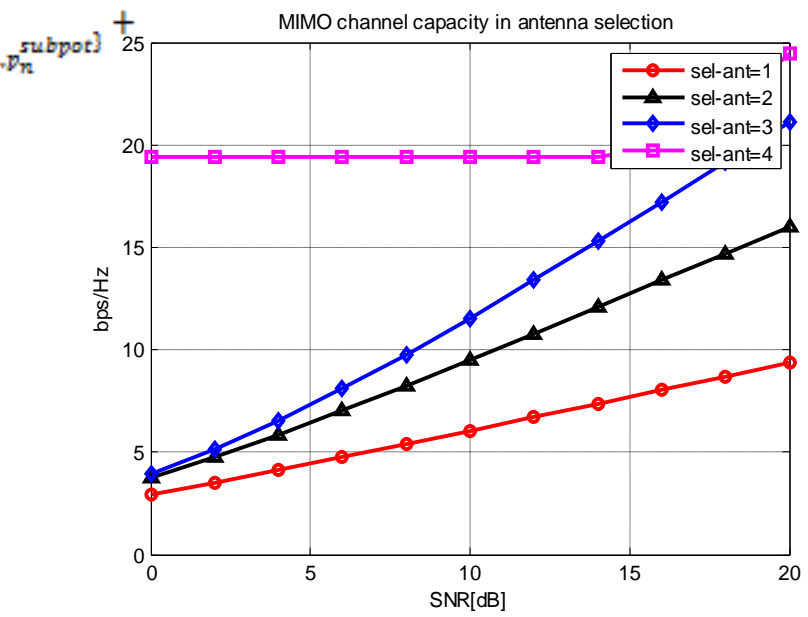

Fig.3: Capacity based Sub-optimal technique for 6X4 MIMO system

Till now Number transmit reception apparatus equivalents to beneficiary receiving wire, however here change the transmitter radio wire is More than get reception apparatus here just radio wire 4 is steady at low SNR and other Antenna 1 to 3 is limit increments regarding SNR as SNR increment the Capacity is increments at higher SNR radio wire 4 is great limit entertainer by watching these chart problematic strategy give the great limit yield in unequal number of transmitter and collector.

\section{CONCLUSION}

We had re-enacted imperfect reception apparatus choice procedures for $4 \mathrm{X} 4$ and $6 \mathrm{X} 4 \mathrm{MIMO}$ framework and it will give a probability of significant gain increment through expanding and diminishing request by climbing or diving determination methodologies as for radio wires. It was seen that the 4X4 MIMO framework at low SNR high limit of about $21 \mathrm{~dB}$ for radio wire 1 and the high limit is $22 \mathrm{~dB}$ is get at $25 \mathrm{~dB}$ SNR. For $6 \mathrm{X} 4 \mathrm{MIMO}$ framework determination of 4 radio wires is taken. As contrast with conventional ideal radio wire determination the multifaceted nature is lessens by choosing the sub set of receiving wires, so sub-ideal method is great perform of limit.

\section{REFERENCES}

1. HaiyangDing, JianhuaGe, DanielB.daCosta, Theodoros, "A Novel Distributed Antenna Selection Scheme for Fixed-Gain Amplify andForward Relaying Systems"IEEE transactions on vehicular technology, vol.61. NO.6.pp.2836-2842 Jul.2012

2. YujiaHu, Xiaofeng Tao, "Secrecy Outage Analysis of Multiuser Diversity with Unequal Average SNR in Transmit Antenna Selection system", IEEE communication letters, vol.10.No.10, pp.1-4, Oct.2004

3. Yuima Hu, Xiao Feng Tao, "Secrecy Outage on Transmit Antenna Selection with weighting errors at maximal-ratio combiners", IEEE communication letters, vol.10.No.10, pp.4-8 Oct.2004

4. Hassan A. "Cross-Layer Based Transmit Antenna Selection for Decision-Feedback Detection in Correlated Rican MIMO Channels", IEEE transactions on wireless Communications, vol. 8, No.4, pp.1677-1682 Apr.2009

5. Qian Ma and CihanTepedelenlio־glu, "Antenna Selection for No coherent Space-Time- Frequency-Coded OFDM'IEEE transactions on vehicular technology, vol. 57, no. 1, pp.615-620, Jan 2008

6. DhananjayA, "Receive Antenna Selection for MIMO Flat-Fading Channels: Theory and Algorithms"IEEE transactions on signal processing, vol.49, NO.10, pp.2687-2696 Oct.2003 
7. Yohan Kim, Student Member, SsangYong Cho, and Dong $\mathrm{Ku} \mathrm{Kim}$, "Low Complexity Antenna Selection Based MIMO Scheduling Algorithms for Uplink Multiuser MIMO/FDD Systemise Publication, pp.1663-1667 2007

8. Michael A.Jensen, and Matthew Lorries, "Efficient capacity-based antenna selection for MIMO systems", IEEE transactions on vehicular technology, vol.54. NO1. Pp.110-116, Jan.2005

9. AlexeiGorokhov, Dhananjay A. Gore, and Arogyaswami, "Receive Antenna Selection for MIMO Spatial Multiplexing: Theory and Algorithms"IEEE Transactions on signal processing, Vol.51, No.11. pp.2796-2805, Nov.2003

10. A.F.Molish and M.Z. Win, "MIMO systems with antenna selection", IEEE Microwave Magazine, vol. 5, pp.46-56, Mar 2004

11. S.M.Alamouti, "A simple transmit diversity technique for wireless communications", IEEE Areas Comm, vol.16, no.8, .pp.1451-1458, Oct.1998.

12. Mahmood "Transmit Antenna Selection for Downlink Transmission in a Massively Distributed Antenna System Using Convex Optimization" (BWCCA) Seven Inter

National Conference Publication (2012)

13. R.W.Heath, Sandhu and A.Paulraj, "Antenna selection for spatial multiplexing systems with linear receivers", IEEE Comm. Letters, Vol5, No.4, pp142-144, April 2001 